\title{
Immunofluorescence Studies on the Expression of the SARS-CoV-2 Receptors in Human Term Placenta
}

\author{
Jürgen Becker ${ }^{\mathrm{a}}$ Danny Qiu $^{\mathrm{a}} \quad$ Walter Baron ${ }^{\mathrm{b}}$ Jörg Wilting ${ }^{\mathrm{a}}$ \\ ${ }^{a}$ Department of Anatomy and Cell Biology, University Medical School Göttingen, Göttingen, Germany; ${ }^{\text {b Department }}$ \\ of Gynecology, Agaplesion Hospital Neu Bethlehem, Göttingen, Germany
}

\section{Keywords}

Placenta - Syncytiotrophoblast · Stromal cells $\cdot$ Hofbauer cells · Endothelial cell · Angiotensin-converting enzyme 2 . TMPRSS2 - Neuropilin 1 - TEK tyrosine kinase - CCBE1 . Vimentin

\begin{abstract}
Until September 2021, the Severe Acute Respiratory Syndrome Coronavirus-2 (SARS-CoV-2; COVID-19) pandemic caused over 217 million infections and over 4.5 million deaths. In pregnant women, the risk factors for the need of intensive care treatment are generally the same as in the overall population. Of note, COVID-19-positive women deliver earlier than COVID-19-negative women, and the risk for severe neonatal and perinatal morbidity and mortality is significantly higher. The probability and pathways of vertical transmission of the virus from the pregnant woman to the fetus are highly controversial. Recent data have shown that 54 (13\%) of 416 neonates born to COVID-19-positive women were infected. Here, we investigated term placentas collected before the SARS-CoV-2 pandemic and studied the main COVID-19 receptors angiotensin-converting enzyme 2 (ACE2), transmembrane protease serine subtype 2
\end{abstract}

(TMPRSS2), as well as neuropilin 1 (NRP1). We performed real-time PCR and immunofluorescence on cryosections in combination with markers for syncytiotrophoblast, endothelial cells, macrophages and stromal cells. The PCR studies showed expression of both the truncated delta form of ACE2, which does not bind the COVID-19 spike protein, and the long form. The ACE2 antibody used does not distinguish between the two forms. We did not observe expression of the canonical SARS-CoV-2 entry machinery on syncytio- and cytotrophoblast. ACE2 and TMPRSS2 are co-expressed in a subpopulation of stromal cells, which in part are CD68-positive macrophages. NRP1 is localized to endothelial cells. In sum, the term placenta is not an organ that directly favors vertical transmission of COVID-19; however, microtraumas and placentitis may weaken its barrier function.

$$
\begin{aligned}
& \text { (C) } 2021 \text { The Author(s). } \\
& \text { Published by S. Karger AG, Basel }
\end{aligned}
$$

\section{Introduction}

As of August 31,2021, the World Health Organization has confirmed over 217 million infections and over 4.5 million deaths caused by Severe Acute Respiratory Syndrome Coronavirus-2 (SARS-CoV-2; COVID-19)
C 2021 The Author(s).

Published by S. Karger AG, Basel

This article is licensed under the Creative Commons Attribution 4.0 International License (CC BY) (http://www.karger.com/Services/ OpenAccessLicense). Usage, derivative works and distribution are permitted provided that proper credit is given to the author and the original publisher.
Correspondence to:

Jörg Wilting, joerg.wilting@ med.uni-goettingen.de 
[https://www.arcgis.com/apps/dashboards / bda7594740fd40299423467b48e9ecf6). Similar to the overall population, in pregnant women, pre-existing comorbidities, high maternal age, and high body mass index are among the risk factors for the need of intensive care treatment [Allotey et al., 2020]. Thereby, vertical transmission to the embryo or fetus is a matter of great general interest. Villar et al. studied vertical transmission of COVID-19 and compared 706 pregnant women with COVID-19 to 1,424 pregnant women without COVID-19. Of note, COVID- $19^{+}$women delivered earlier than COVID-19- women, and the ones with symptoms 1.5 days earlier than the ones without symptoms, after approx. 30 weeks' gestation [Villar et al., 2021]. The risk for severe neonatal and perinatal morbidity and mortality was significantly higher in the group of COVID- $19^{+}$ women. 416 neonates born to COVID- $19^{+}$women were tested for the virus, and of these, 54 (13\%) were COVID- $19^{+}$. Thereby, cesarean delivery may have increased the risk for neonates to test positively (RR, 2.15; 95\% CI, 1.18-3.91), and Villar et al. emphasize that contamination during cesarian resection has to be taken into consideration. In a previous review on 324 pregnant women with COVID-19, only 3 of 155 neonates tested were COVID-19+ [Juan et al., 2020]. In a living systematic review (last update Nov. 29, 2020) it is noted that "existing evidence has not identified major risks of complications in babies born to mothers with COVID-19" [Yap et al., 2020]. More recent studies confirmed increased risks of preterm delivery and maternal mortality caused by COVID-19 infection [Mullins et al., 2021]. Transfer of antiCOVID-19 IgG from mother to fetus is very likely, but IgM antibodies, which are unlikely to pass the placental barrier, have also been detected in some newborns [Zeng et al., 2020; Dong et al., 2020].

Considering how close the connection between mother and fetus is, vertical transmission of COVID-19 appears to be relatively low. There are, however, a number of studies that point to the existence and significance of COVID-19 receptors in placenta. Immunoperoxidase studies showed focal expression of the main COVID-19 receptor angiotensin-converting enzyme-2 (ACE2) on placental syncytiotrophoblast [Hikmet et al., 2020], immediately at the feto-maternal interface. Additionally, with analyses of the GEO scRNASeq database, Lü et al. found expression of ACE2 in 9 out of 9,852 cells derived from one placenta, which were also GATA3-positive and therefore classified as trophoblast cells (not differentiating between cyto- and syncytiotrophoblast) [Lü et al., 2020]. The authors took this as an indication that mater-

SARS-CoV-2 Receptors in Human Term Placenta nal COVID-19 may directly infect trophoblast cells. Studying single-cell and single-nuclear RNA-sequencing data, Pique-Regi et al. [Pique-Regi et al., 2020] concluded that the number of placenta cells coexpressing ACE2 and the serine protease TMPRSS2, which is widely accepted as the most important activator for cell entry of SARSCoV-2 [Hoffmann et al., 2020], is negligible, making vertical transmission highly unlikely. In contrast, with paraffin histology, expression of ACE2 protein and SARSCoV-2 spike RNA in multiple parts of placenta and umbilical cord have been described [Verma et al., 2021]. However, the effects of boiling tissues in a microwave, as in these studies [Verma et al., 2021], are not necessarily predictable.

Here, we investigated term placentas collected in 2015 before SARS-CoV-2 pandemic and studied ACE2 and TMPRSS2 by real-time PCR (qPCR) and Western blot. We then performed immunofluorescence (IF) on cryosections to study the cellular localization of these molecules in addition to neuropilin-1 (NRP1), which can assist SARS-CoV-2 infection [Li and Buck, 2021], in combination with markers for syncytiotrophoblast, endothelial cells, macrophages, and stromal cells. We did not observe evidence for the expression of SARS-CoV-2 entry molecules on syncytio- and cytotrophoblast. ACE2 and TMPRSS2 are found in stromal cells, which in part are CD68-positive macrophages. Our qPCR analyses showed expression of the long and the truncated (delta) isoform of ACE2, which does not bind to the viral spike protein [Onabajo et al., 2020]. Under physiological conditions, the term placenta appears to provide a barrier against SARS-CoV-2 infection.

\section{Materials and Methods}

\section{Tissues and Cells}

Six term placentas were collected after vaginal births of healthy children with the informed, written consent of the mothers. Specimens were collected in 2015 clearly before the start of the COVID-19 pandemic, and stored at $-80^{\circ} \mathrm{C}$. Specimens were rinsed in phosphate-buffered saline (PBS) and freshly frozen for Western blot analysis and real-time PCR, or fixed for histology and immunohistology in $4 \%$ paraformaldehyde overnight. The studies were approved by the ethics committee of the University Medical Hospital Göttingen, UMG (application no. 18/1/18), and methods were performed in accordance with the relevant guidelines and regulations. For comparative Western blotting and qPCR, human lymphatic endothelial cells were purchased from PromoCell (Heidelberg, Germany), cultured in LEC medium and checked for purity, as described recently [Blesinger et al., 2018]. Various human tumor cell lines were cultured under standard conditions as described before [Becker et al., 2010]. 
Table 1. Antibodies used in this study

\begin{tabular}{|c|c|c|c|}
\hline & Dilution & Manufacturer & Lot \\
\hline \multicolumn{4}{|l|}{ Primary antibody } \\
\hline$\beta$-Catenin, mouse-anti-human & $1: 500$ & Biosciences, Franklin Lakes, USA & 610153 \\
\hline CCBE1, rabbit-anti-human & $1: 500$ & Sigma-Aldrich, Munich, Germany & R38605 \\
\hline CD68, mouse-anti-human & $1: 10$ & Dako, Jena, Germany & 20055015 \\
\hline ACE-2, mouse-anti-human & $1: 100$ & $\begin{array}{l}\text { ReliaTech, Wolfenbüttel } \\
\text { Germany }\end{array}$ & $11 \mathrm{~A} 31$ \\
\hline TMPRSS2, rabbit-anti-human & $1: 100$ & Abcam, Cambridge, UK & GR3343160-1 \\
\hline NRP1, rabbit-anti-human & $1: 100$ & $\begin{array}{l}\text { ReliaTech, Wolfenbüttel } \\
\text { Germany }\end{array}$ & 1403R16 \\
\hline TIE2, mouse-anti-human & $1: 100$ & $\begin{array}{l}\text { ReliaTech, Wolfenbüttel } \\
\text { Germany }\end{array}$ & 0512R09 \\
\hline $\begin{array}{l}\text { Vimentin, mouse-anti-human } \\
\text { Secondary antibody }\end{array}$ & $1: 10$ & Dako, Jena, Germany & 20035381 \\
\hline IgG H + L, goat-anti-rabbit Alexa 488 & $1: 200$ & Invitrogen, Waltham, USA & A11008 \\
\hline IgG H + L, goat-anti-mouse Alexa 594 & $1: 200$ & Invitrogen, Waltham, USA & A11008 \\
\hline IgG1, goat-anti-mouse Alexa 488 & $1: 200$ & Invitrogen, Waltham, USA & A21121 \\
\hline IgG2a, goat-anti-mouse Alexa 594 & $1: 200$ & Invitrogen, Waltham, USA & 2044860 \\
\hline
\end{tabular}

Table 2. Primers used for $q P C R$

\begin{tabular}{lll}
\hline Genes & Primer name & Sequence \\
\hline ACE2 & ACE2_fwd & AGAAAGCAGTCTGCCATCCC \\
& ACE2_rev & GCTGTCAGGAAGTCGTCCAT \\
\hline TMPRSS2 & TMPRSS2_fwd & ATACAAGCTGGGGTTCTGGC \\
& TMPRSS2_rev & AGACCATGTGGATTAGCCGT \\
\hline 3-Actin & Actin_fwd & ATTGGCAATGAGCGGTTC \\
& Actin_rev & TGAAGGTATTCGTGGATGC \\
\hline ACE2 total & totACE2_fwd & TGGGACTCTGCCATT TACTTAC \\
\hline ACE2 long isoform & totACE2_rev & CCCAACTATCTCTCG CTTCATC \\
\hline ACE2 short isoform & lonACE2_fwd & CAAGAGCAAACGGTTGAACAC \\
& lonACE2_rev & CCAGAGCCTCTCATT GTAGTCT \\
\hline
\end{tabular}

IF Studies

Specimens were rinsed in PBS, transferred into 10 and $30 \%$ sucrose in PBS, and embedded in tissue freeze medium (Tissue Tek, Sakura Finetek Zoeterwoude, NL). Sections of $12 \mu \mathrm{m}$ were incubated with the primary and secondary antibodies listed in Table 1. Sections were counter-stained with DAPI, and mounted under coverslips with Fluoromount-G (Southern Biotechnology, Birmingham, AL, USA). Photos were taken with AxioImagerZ1 (Zeiss, Göttingen, Germany).

Real-Time RT-PCR

Real-time RT-PCR (qPCR) was performed as described [Becker et al., 2021]. Primers are listed in Table 2.

\section{Results}

With qPCR, we found clear signals for both ACE2 and TMPRSS2 in term placenta. ACE2 exists in various isoforms ranging from $805 \mathrm{aa}$ ( $92.5 \mathrm{kDa}$; canonical isoform) to 459 aa $(52.7 \mathrm{kDa}$; short, deltaACE2; see: https://www. uniprot.org/uniprot/Q9BYF1). With specific primers, we obtained signals for both the long and, to a lesser extent, the short/delta variant of ACE2 (data not shown). Our IF studies of ACE2 in term placenta were performed with an antibody raised against aa 19 to aa 740 of ACE2, and therefore not distinguishing between the short and the 

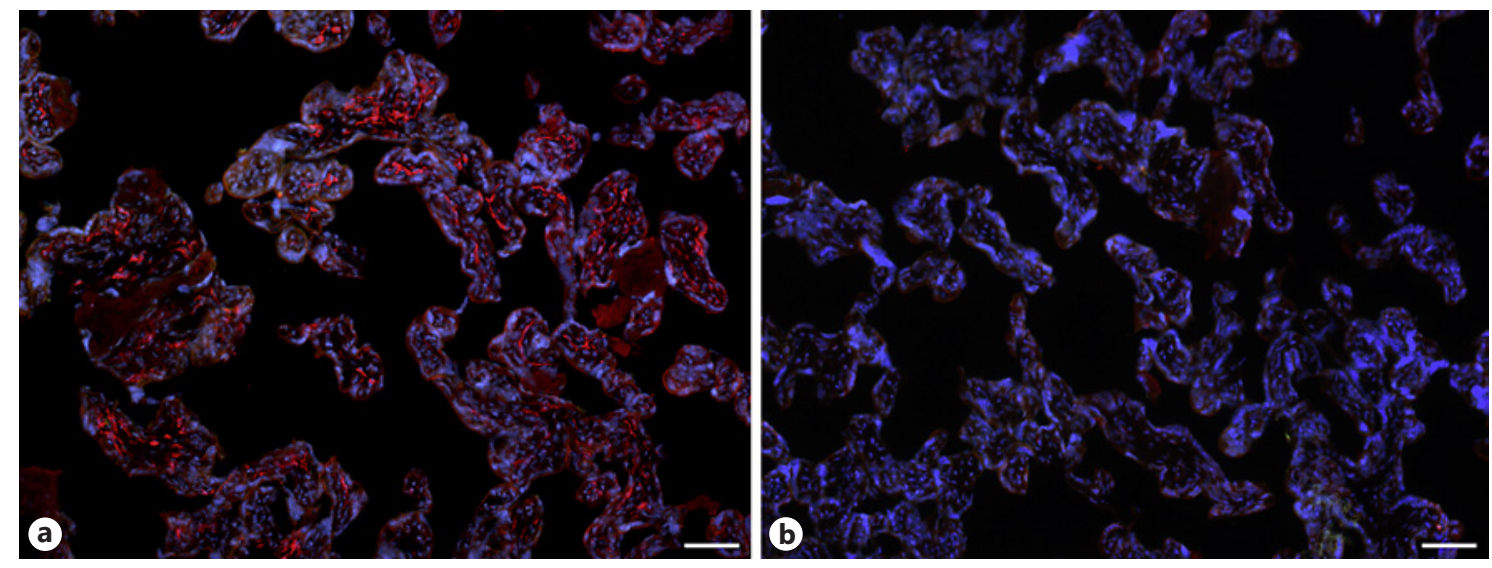

Fig. 1. a Immunofluorescence picture showing ACE2 (red) in term placenta. b Control without primary antibody. ACE2 is found in stromal cells. Nuclei are stained blue with DAPI. Bar $=80 \mu \mathrm{m}$.

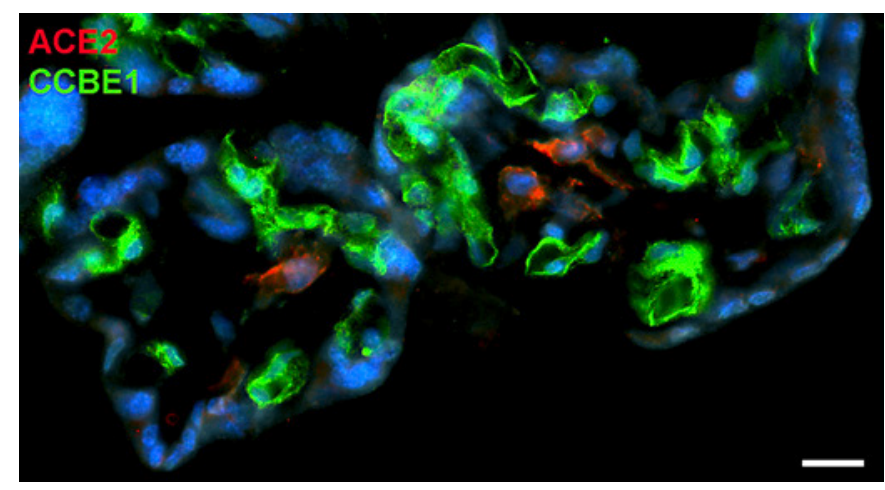

Fig. 2. Immunofluorescence picture showing ACE2 (red) and CCBE1 (green) in term placenta. CCBE1 demarcates the capillaries and ACE2 stromal cells. Nuclei are stained blue with DAPI. Bar $=20 \mu \mathrm{m}$.

long splice variant. Our studies revealed a signal in the stromal compartment of the villi (Fig. 1). To further identify the cells, we performed double IF with various markers. As shown previously [Becker et al., 2020], collagenand calcium-binding EGF domain 1 (CCBE1) is a marker of villous endothelial cells. We observed ACE2 in stromal cells, not in endothelial cells (Fig. 2). The cell junction molecule $\beta$-catenin, a key mediator of the WNT-signaling pathway, was expressed at the basal side of the syncytiotrophoblast, and again, ACE2 was found in stromal cells (Fig. 3). We then performed double-staining with antibodies against ACE2 and the macrophage marker CD68 and found double-positive cells (Fig. 4).

SARS-CoV-2 Receptors in Human Term Placenta

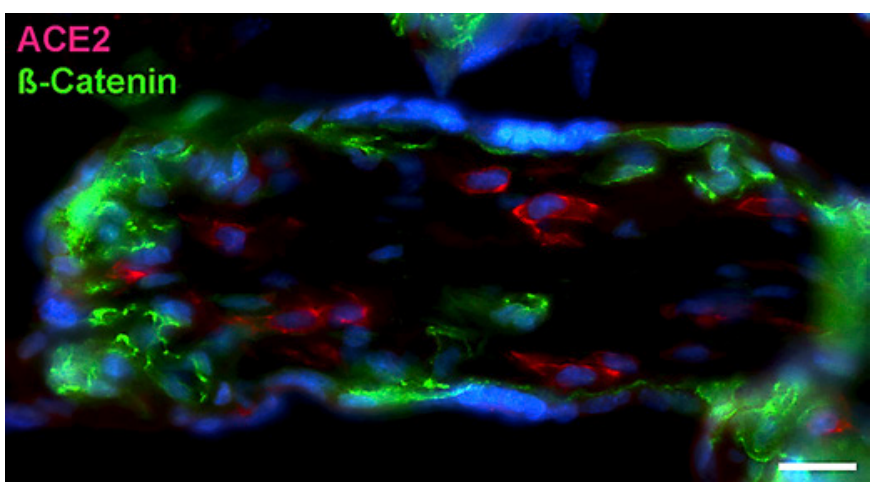

Fig. 3. Immunofluorescence picture showing ACE2 (red) and $\beta$-catenin (green) in term placenta. $\beta$-Catenin demarcates mainly the basal aspect of the syncytiotrophoblast. ACE2 is found in stromal cells. Nuclei are stained blue with DAPI. Bar $=25 \mu \mathrm{m}$.

Next, staining with antibodies against TMPRSS2 also revealed positive cells in the stroma. Double-staining with the mesenchymal marker vimentin showed that there was almost no overlap of the two signals (Fig. 5). Double staining with antibodies against the endothelial marker TIE2 (TEK tyrosine kinase) confirmed TMPRSS2 expression in stromal cells, not in endothelial cells (Fig. 6). Double staining against TMPRSS2 and ACE2 revealed cells in the stroma, which expressed TMPRSS2 and ACE2 cells (Fig. 7). Furthermore, double-staining for TMPRSS2 and CD68 revealed co-expression in macrophages (Fig. 8). Staining for NRP1 revealed a strong signal on endothelial cells, but no signal at the apical surface of the syncytiotrophoblast (Fig. 9).

Cells Tissues Organs 2023;212:138-146 


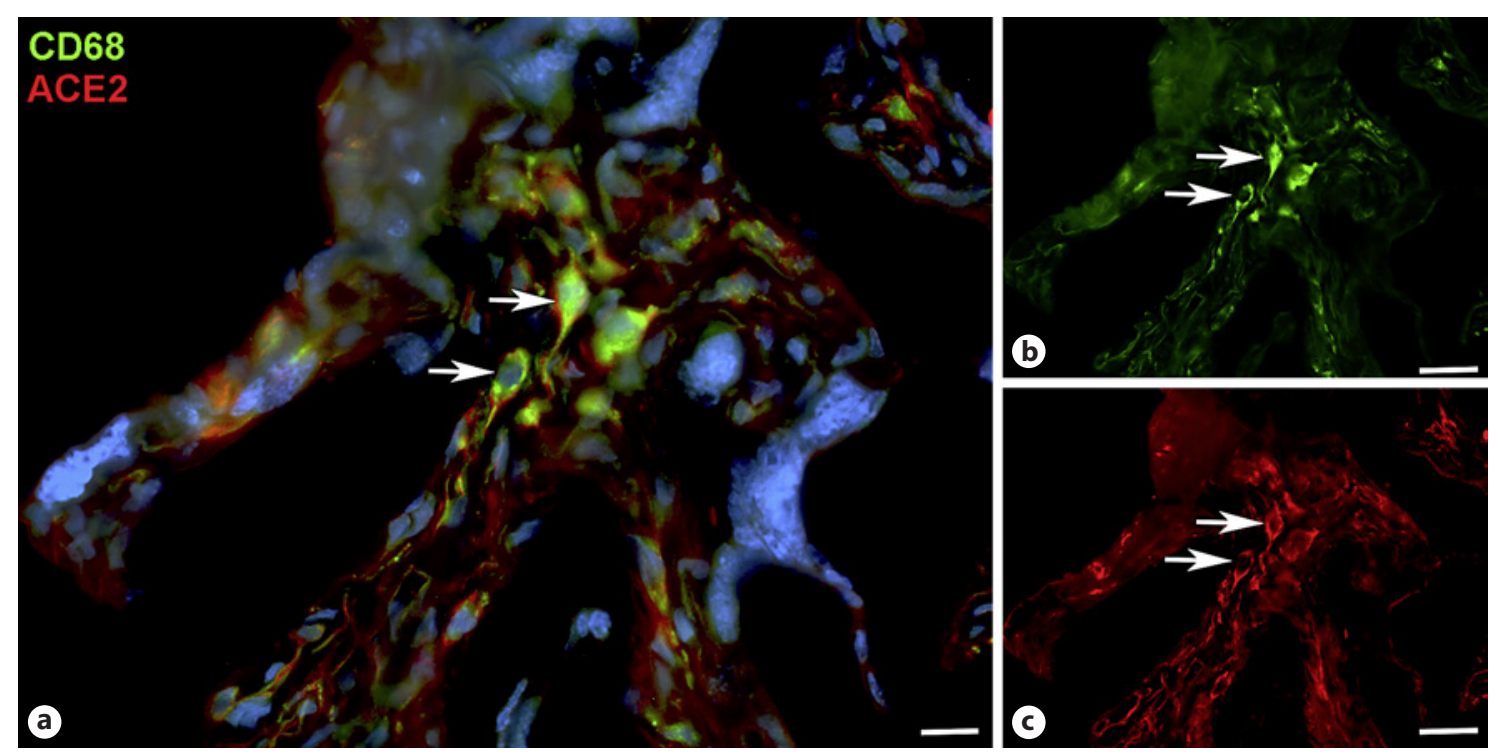

Fig. 4. Immunofluorescence picture showing ACE2 (red) and macrophage marker CD68 (green) in term placenta. a Merged picture; $\mathbf{b}$ and $\mathbf{c}$ show each channel separately. A subpopulation of ACE2+ cells can be identified as macrophages (arrows). Nuclei are stained blue with DAPI. Bar $=20 \mu \mathrm{m}$ in A, and $40 \mu \mathrm{m}$ in B, C.

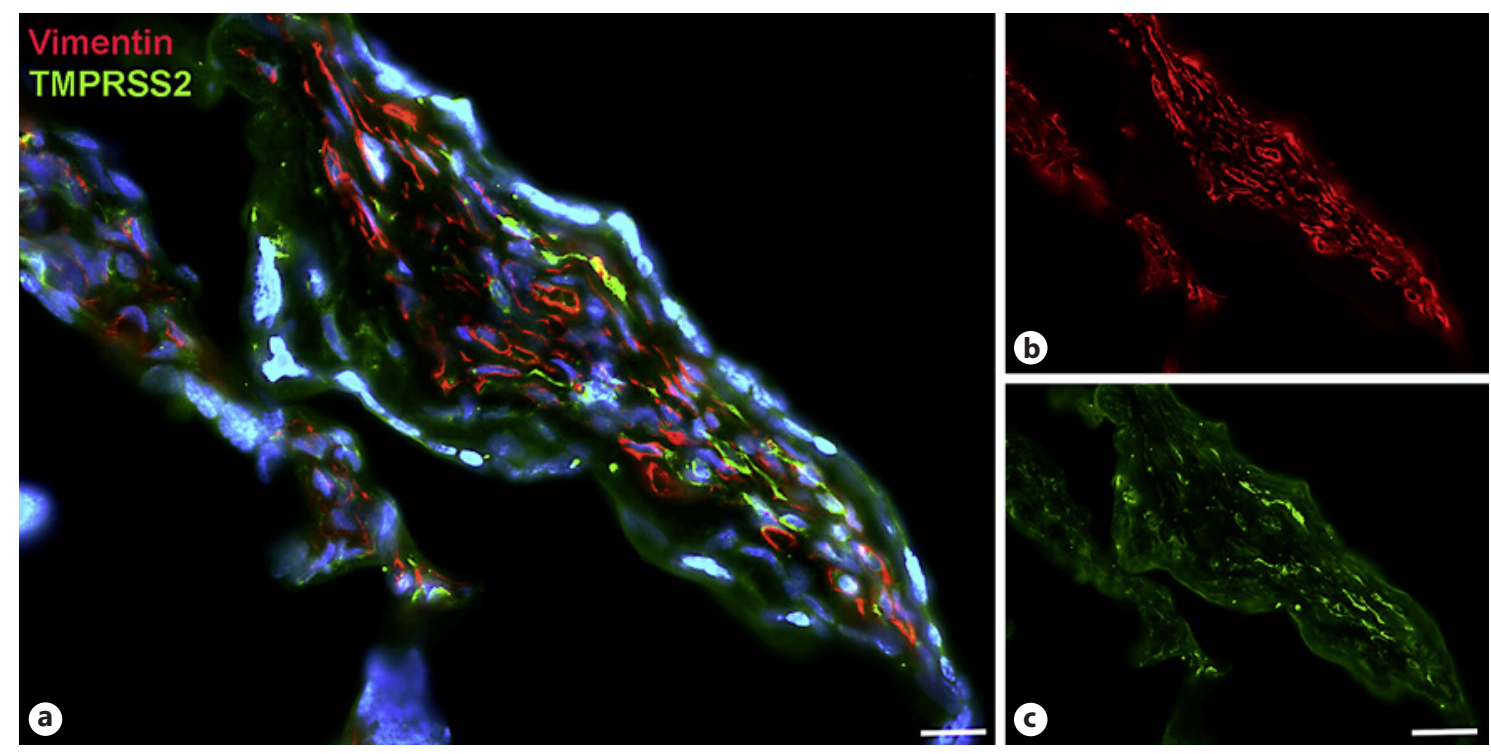

Fig. 5. Immunofluorescence picture showing TMPRSS2 (green) and mesenchymal marker vimentin (red) in term placenta. a Merged picture; $\mathbf{b}$ and $\mathbf{c}$ show each channel separately. The two signals are found in stromal cells, but there is hardly any overlap. Nuclei are stained blue with DAPI. Bar $=20 \mu \mathrm{m}$ in $\mathbf{a}$ and $40 \mu \mathrm{m}$ in $\mathbf{b}$ and $\mathbf{c}$.

\section{Discussion}

We studied the expression of COVID19 receptors in term placenta. We observed immunopositivity for ACE2 and TMPRSS2 in stromal cells. A subpopulation of the cells stained positive for $\mathrm{CD} 68$, and can therefore be designated as macrophages/Hofbauer cells. This is in line with the finding of SARS-CoV-2 RNA in $\mathrm{CD} 14^{+}$mononuclear cells in placental villi of COVID- $19^{+}$women [Facchetti et al., 2020]. By qPCR, we observed expression 
Fig. 6. Immunofluorescence picture showing endothelial cell marker TIE2 (green) and TMPRSS2 (red) in term placenta. There is no overlap of the signals. Nuclei are stained blue with DAPI. Bar $=20 \mu \mathrm{m}$.
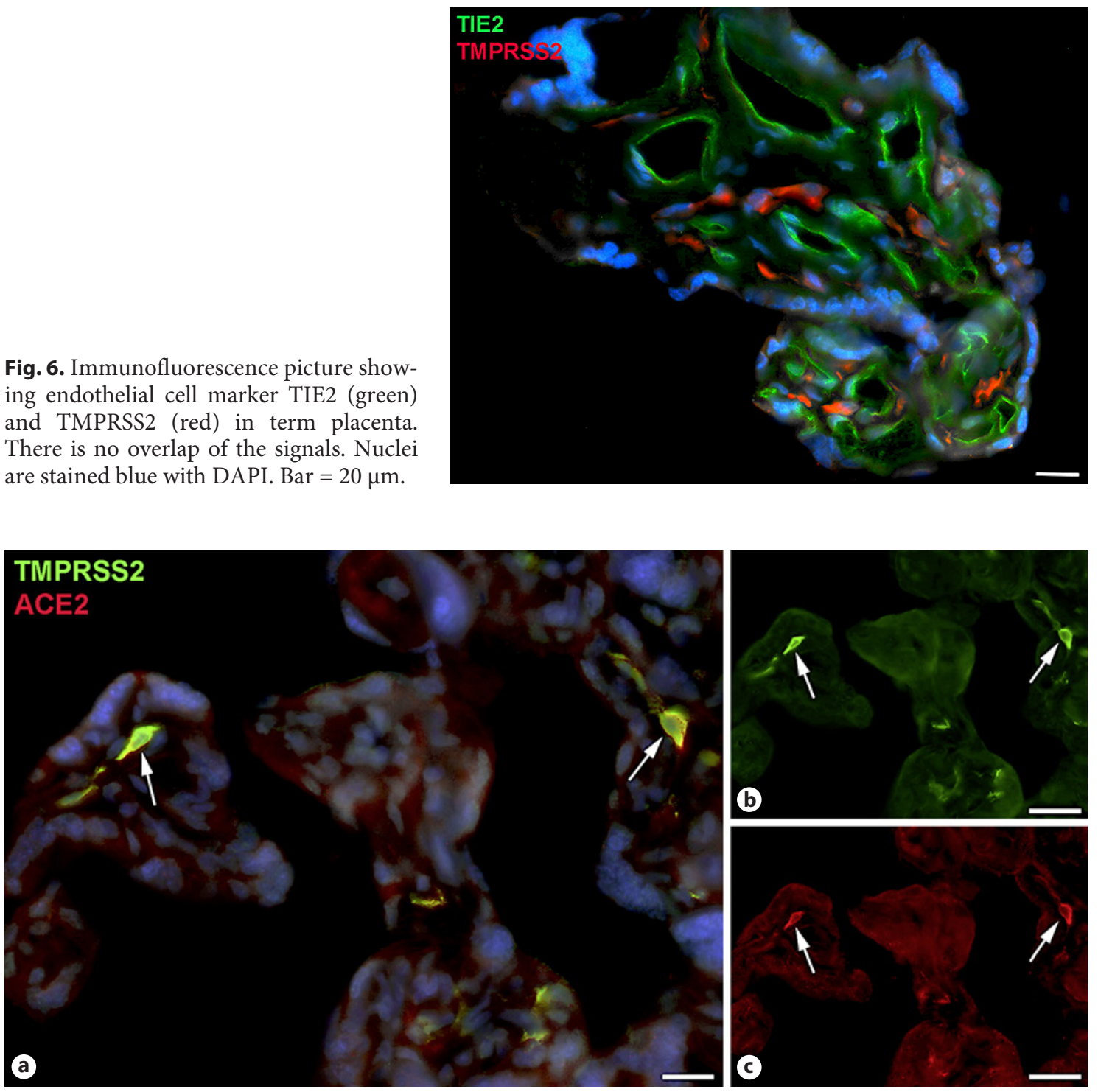

Fig. 7. Immunofluorescence picture showing TMPRSS2 (green) and ACE2 (red) in term placenta. a Merged picture; $\mathbf{b}$ and $\mathbf{c}$ show each channel separately. A number of TMPRSS2+ stromal cells also express ACE2 (arrows). Nuclei are stained blue with DAPI. Bar $=20 \mu \mathrm{m}$ in $\mathbf{a}$ and $40 \mu \mathrm{m}$ in $\mathbf{b}$ and $\mathbf{c}$.

of the long and the short (delta) isoform of ACE2. It was shown recently that the truncated isoform can be induced by interferons and rhinoviruses, but not by SARS-CoV-2 infection [Onabajo et al., 2020; Blume et al., 2021]. These studies also showed that delta-ACE2 does not bind the COVID-19 spike protein and does not possess carboxypeptidase activity. As noted above, the antibody used does not distinguish between the truncated and the long form of ACE2. Nevertheless, expression in stromal cells, which are protected by the placental barrier, suggests that vertical transmission may be unlikely under physiological conditions. The specimens studied here were collected before the start of the COVID-19 pandemic from healthy women. We are therefore confident that the expression pattern described here is the physiological one. Its alteration during infection, however, cannot be ruled out.

We found expression of NRP1, a typical endothelial receptor [Shraga-Heled et al., 2007], in endothelial cells of placental vessels. None of the three investigated COVID19 receptors/coreceptors was found on the apical 


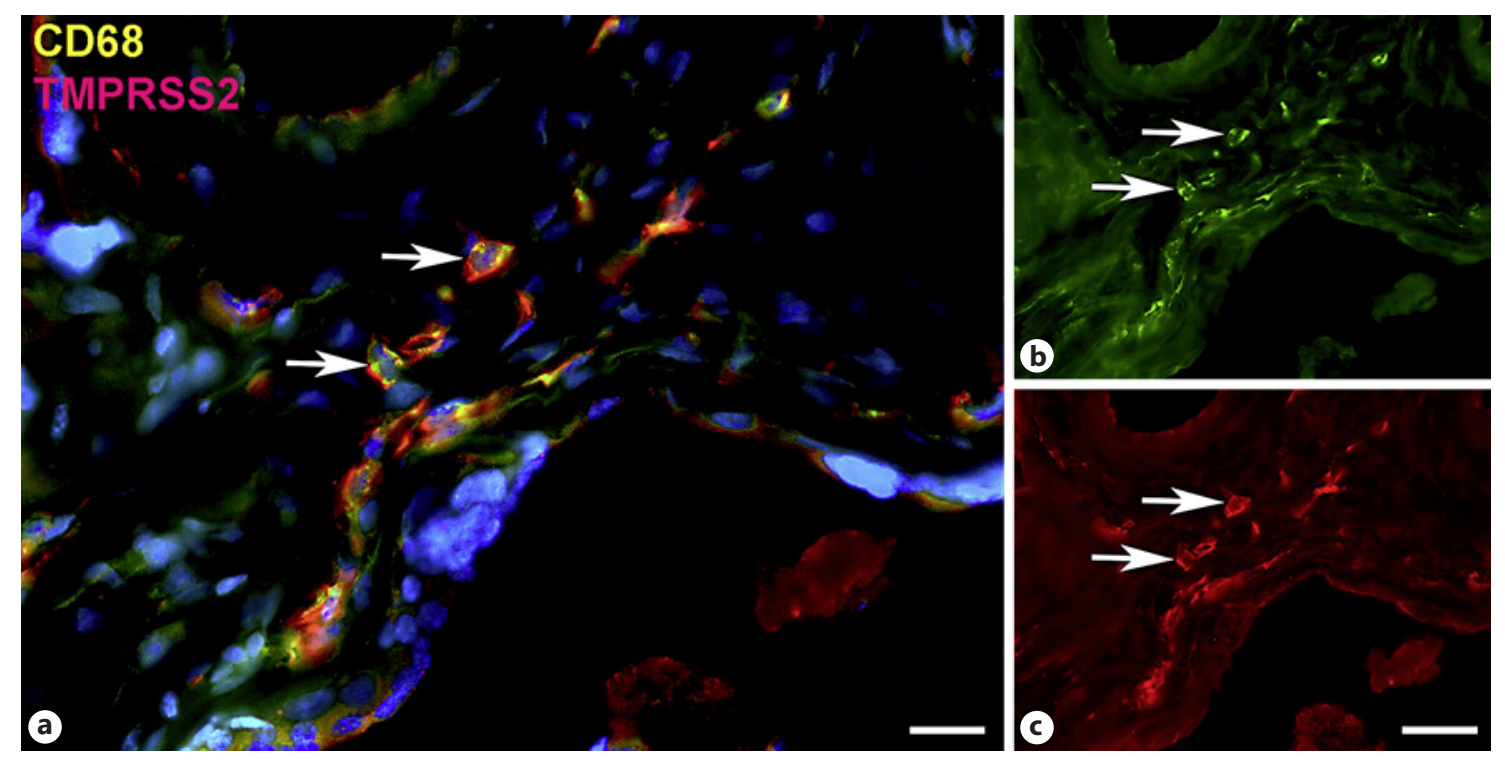

Fig. 8. Immunofluorescence picture showing TMPRSS2 (red) and macrophage marker CD68 (green) in term placenta. a Merged picture; $\mathbf{b}$ and $\mathbf{c}$ show each channel separately. A subpopulation of TMPRSS2+ cells can be identified as macrophages (arrows). Nuclei are stained blue with DAPI. Bar $=20 \mu \mathrm{m}$ in $\mathbf{a}$ and $40 \mu \mathrm{m}$ in $\mathbf{b}$ and $\mathbf{c}$.
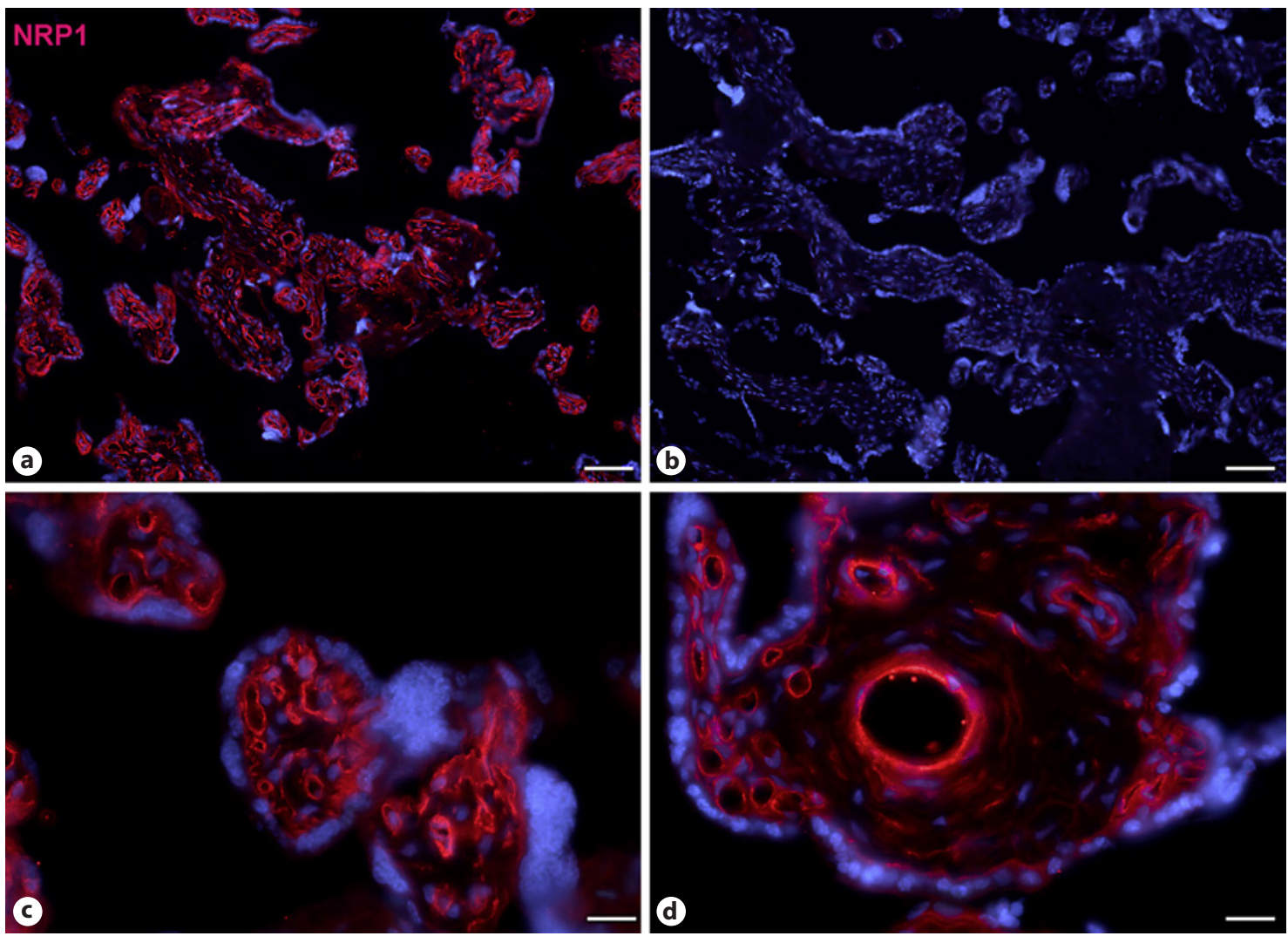

Fig. 9. Immunofluorescence picture showing NRP1 (red) in term placenta. High NRP1 expression is found in endothelial cells. Nuclei are stained blue with DAPI. b Negative control without primary antibody. Bar $=80 \mu \mathrm{m}$ in $\mathbf{a}$ and $\mathbf{b}$, and $20 \mu \mathrm{m}$ in $\mathbf{c}$ and $\mathbf{d}$. 
surface of the syncytiotrophoblast, which is the only physiological contact site for maternal blood in term placenta. Of note, we also studied ACE2 expression on paraffin sections with antigen retrieval methods (data not shown). This produced background staining, e.g. in nuclei in control sections, which appeared to be enhanced by the primary antibody.

With scRNASeq, expression of ACE2 was found in 9 out of 9,852 placenta-derived cells. These cells were also GATA3-positive and therefore classified as trophoblast cells; leaving open, which type of trophoblast cell they were exactly [Lü et al., 2020]. Single-cell RNA analyses of syncytiotrophoblast are, for obvious reasons, difficult to interpret. Also, the localization of the protein in the cell (apical vs. basal) must be determined. There are a number of studies which argue against vertical transmission during pregnancy, while others show ACE2 immunopositivity in syncytiotrophoblast (an overview of the literature can be found, e.g. in [Verma et al., 2021; Linehan et al., 2021]. Thereby, ACE2 expression was usually shown in placentas from COVID- $19^{+}$women, but coexpression of TMPRSS2 was not detected [Taglauer et al., 2020]. SARS$\mathrm{CoV}-2$ infection in syncytiotrophoblast was detected, e.g. with immunostaining against the viral nucleocapsid or spike protein [Facchetti et al., 2020; Hsu et al., 2021]. However, COVID-19 positivity in the syncytiotrophoblast does not necessarily correlate with viral infection of the fetus [Penfield et al., 2020]. In 2 out of 22 neonates described by Patane et al., COVID-19-positivity was observed in syncytiotrophoblast in addition to histiocytic intervillositis, associated with macrophages in both the intervillous and villous spaces [Patane et al., 2020].

\section{Conclusion}

Recent data provide evidence that in a certain number of neonates (around 13\%) born to COVID- $19^{+}$women vertical transmission does occur [Villar et al., 2021]. A very recent study confirms the high risks of SARS-CoV-2 infection for the pregnant women and their fetuses, but it also emphasizes that "there were no additional adverse neonatal outcomes, other than those related to preterm delivery" [Gurol-Urganci et al., 2021]. Our data on healthy term placenta seem to show that the placenta provides a barrier against vertical transmission of COVID- 19 . The main receptors for viral entry, TMPRSS2 and ACE2, are not detectable by IF on the apical surface of the syncytiotrophoblast. Stromal cells, which in part are CD68 ${ }^{+}$ Hofbauer cells, seem to be most susceptible for CO-
VID-19 infection by expressing TMPRSS2 and ACE2. Virus propagation via circulating mononuclear cells was proposed earlier [Facchetti et al., 2020]. Microtrauma during placental development (which is the most likely reason for fibrinoid deposits), as well as placentitis [Linehan et al., 2021], and probably cesarian resection [Villar et al., 2021] may also be routes for vertical transmission of SARS-CoV-2. However, further viral receptors and coreceptors, such as TMPRSS4 [Zang et al., 2020], must be taken into consideration.

\section{Acknowledgements}

We thank Ch. Zelent, S. Schwoch, and B. Manshausen for their expert technical assistance.

\section{Statement of Ethics}

Placentas were collected after vaginal births of healthy children with the informed, written consent of the mothers. The studies were approved by the ethics committee of the University Medical Hospital Göttingen, UMG (application No.: 18/1/18).

\section{Conflict of Interest Statement}

The authors declare that they have no conflicts of interest related to the research.

\section{Funding Sources}

We did not receive specific funding to conduct this study.

\section{Author Contributions}

Jürgen Becker: data curation, supervision. Danny Qiu: data acquisition, writing - draft. Walter Baron: material acquisition and patient education. Jörg Wilting: conceptualization, data curation, supervision, writing - review and editing.

\section{Data Availability Statement}

All immunofluorescence data are included in the article. Realtime PCR data will be presented upon request. 


\section{References}

Allotey J, Stallings E, Bonet M, Yap M, Chatterjee $\mathrm{S}$, Kew T, et al. Clinical manifestations, risk factors, and maternal and perinatal outcomes of coronavirus disease 2019 in pregnancy: living systematic review and meta-analysis. BMJ. 2020;370:m3320.

Becker J, Wang B, Pavlakovic H, Buttler K, Wilting J. Homeobox Transcription Factor Prox1 in Sympathetic Ganglia of Vertebrate Embryos: Correlation With Human Stage 4s Neuroblastoma. Pediatr Res. 2010;68:1127.

Becker J, Tchagou Tchangou GE, Schmidt S, Zelent C, Kahl F, Wilting J. Absence of lymphatic vessels in term placenta. BMC Pregnancy Childbirth. 2020;20:380.

Becker J, Schwoch S, Zelent C, Sitte M, Salinas G, Wilting J. Transcriptome Analysis of Hypoxic Lymphatic Endothelial Cells Indicates Their Potential to Contribute to Extracellular Matrix Rearrangement. Cells. 2021;10(5).

Blesinger H, Kaulfuß S, Aung T, Schwoch S, Prantl L, Rößler J, et al. PIK3CA mutations are specifically localized to lymphatic endothelial cells of lymphatic malformations. PLoS One. 2018;13:e200343.

Blume C, Jackson CL, Spalluto CM, Legebeke J, Nazlamova L, Conforti F, et al. A novel ACE2 isoform is expressed in human respiratory epithelia and is upregulated in response to interferons and RNA respiratory virus infection. Nat Genet. 2021;53:205-14.

Dong L, Tian J, He S, Zhu C, Wang J, Liu C, et al. Possible Vertical Transmission of SARSCoV-2 From an Infected Mother to Her Newborn. JAMA. 2020. [cited 21 May 2021].

Facchetti F, Bugatti M, Drera E, Tripodo C, Sartori E, Cancila V, et al. SARS-CoV2 vertical transmission with adverse effects on the newborn revealed through integrated immunohistochemical, electron microscopy and molecular analyses of Placenta. EBioMedicine. 2020;59.

Gurol-Urganci I, Jardine JE, Carroll F, Draycott T, Dunn G, Fremeaux A, et al. Maternal and perinatal outcomes of pregnant women with SARS-CoV-2 infection at the time of birth in England: national cohort study. Am J Obstet Gynecol. 2021;0.
Hikmet F, Méar L, Edvinsson Å, Micke P, Uhlén $M$, Lindskog $C$. The protein expression profile of ACE2 in human tissues. Mol Syst Biol. 2020;16:e9610.

Hoffmann M, Kleine-Weber H, Schroeder S, Krüger N, Herrler T, Erichsen S, et al. SARSCoV-2 Cell Entry Depends on ACE2 and TMPRSS2 and Is Blocked by a Clinically Proven Protease Inhibitor. Cell. 2020;181:271-80.e8.

Hsu AL, Guan M, Johannesen E, Stephens AJ, Khaleel N, Kagan N, et al. Placental SARSCoV-2 in a pregnant woman with mild COVID-19 disease. J Med Virol. 2021;93:103844.

Juan J, Gil MM, Rong Z, Zhang Y, Yang H, Poon LC. Effect of coronavirus disease 2019 (COVID-19) on maternal, perinatal and neonatal outcome: systematic review. Ultrasound $\mathrm{Ob}$ stet Gynecol. 2020;56:15-27.

Li Z-L, Buck M. Neuropilin-1 Assists SARSCoV-2 Infection by Stimulating the Separation of Spike Protein Domains S1 and S2. bioRxiv. 2021.

Linehan L, O'Donoghue K, Dineen S, White J, Higgins JR, Fitzgerald B. SARS-CoV-2 placentitis: An uncommon complication of maternal COVID-19. Placenta. 2021;104:261-6.

Lü M, Qiu L, Jia G, Guo R, Leng Q. Single-cell expression profiles of ACE2 and TMPRSS2 reveals potential vertical transmission and fetus infection of SARS-CoV-2. Aging (Albany NY). 2020;12:19880-97.

Mullins E, Hudak ML, Banerjee J, Getzlaff T, Townson J, Barnette K, et al. Pregnancy and neonatal outcomes of COVID-19: coreporting of common outcomes from PAN-COVID and AAP-SONPM registries. Ultrasound $\mathrm{Ob}-$ stet Gynecol. 2021;57:573-81.

Onabajo OO, Banday AR, Stanifer ML, Yan W, Obajemu A, Santer DM, et al. Interferons and viruses induce a novel truncated ACE2 isoform and not the full-length SARS-CoV-2 receptor. Nat Genet. 2020;52:1283-93.

Patanè L, Morotti D, Giunta MR, Sigismondi C, Piccoli MG, Frigerio L, et al. Vertical transmission of coronavirus disease 2019: severe acute respiratory syndrome coronavirus 2 RNA on the fetal side of the placenta in pregnancies with coronavirus disease 2019-positive mothers and neonates at birth. Am J Obstet Gynecol MFM. 2020;2:100145.
Penfield CA, Brubaker SG, Limaye MA, Lighter J, Ratner AJ, Thomas KM, et al. Detection of severe acute respiratory syndrome coronavirus 2 in placental and fetal membrane samples. Am J Obstet Gynecol MFM. 2020;2:100133.

Pique-Regi R, Romero R, Tarca AL, Luca F, Xu Y, Alazizi A, et al. Does the human placenta express the canonical cell entry mediators for SARS-CoV-2? Parker SC. Elife. 2020 Jul;9: e58716.

Shraga-Heled N, Kessler O, Prahst C, Kroll J, Augustin H, Neufeld G. Neuropilin-1 and neuropilin-2 enhance VEGF121 stimulated signal transduction by the VEGFR-2 receptor. FASEB J. 2007;21:915-26.

Taglauer E, Benarroch Y, Rop K, Barnett E, Sabharwal V, Yarrington C, et al. Consistent localization of SARS-CoV-2 spike glycoprotein and ACE2 over TMPRSS2 predominance in placental villi of 15 COVID-19 positive maternal-fetal dyads. Placenta. 2020;100:69-74.

Verma S, Joshi CS, Silverstein RB, He M, Carter EB, Mysorekar IU. SARS-CoV-2 colonization of maternal and fetal cells of the human placenta promotes alteration of local renin-angiotensin system. Med. 2021;2(5):575-90.

Villar J, Ariff S, Gunier RB, Thiruvengadam R, Rauch S, Kholin A, et al. Maternal and neonatal morbidity and mortality among pregnant women with and without COVID-19 infection: The INTERCOVID Multinational Cohort Study. JAMA Pediatr. 2021.

Yap M, Debenham L, Kew T, Chatterjee SR, Allotey J, Stallings E, et al. Clinical manifestations, prevalence, risk factors, outcomes, transmission, diagnosis and treatment of COVID-19 in pregnancy and postpartum: a living systematic review protocol. BMJ Open. 2020;10:e041868.

Zang R, Gomez Castro MF, McCune BT, Zeng Q, Rothlauf PW, Sonnek NM, et al. TMPRSS2 and TMPRSS4 promote SARS-CoV-2 infection of human small intestinal enterocytes. Sci Immunol. 2020 May;5(47):eabc3582.

Zeng H, Xu C, Fan J, Tang Y, Deng Q, Zhang W, et al. Antibodies in infants born to mothers with COVID-19 pneumonia. JAMA. 2020; 323:1848-9. 